Received: 15 January 2019

Accepted: 26 July 2019

Published online: 12 August 2019

\title{
The large repertoire of conifer NLR resistance genes includes drought responsive and highly diversified
} RNLS

\begin{abstract}
Cyril Van Ghelder ${ }^{1,2}$, Geneviève J. Parent $\mathbb{1}^{2,3}$, Philippe Rigault ${ }^{4,5}$, Julien Prunier ${ }^{3}$, Isabelle Giguère ${ }^{3}$, Sébastien Caron ${ }^{4}$, Juliana Stival Sena ${ }^{6}$, Annie Deslauriers $\mathbb{B}^{7}$, Jean Bousquet ${ }^{3,8}$, Daniel Esmenjaud ${ }^{1}$ \& John MacKay ${ }^{2,3}$
\end{abstract}

The NLRs or NBS-LRRs (nucleotide-binding, leucine-rich-repeat) form the largest resistance gene family in plants, with lineage-specific contingents of TNL, CNL and RNL subfamilies and a central role in resilience to stress. The origin, evolution and distribution of NLR sequences has been unclear owing in part to the variable size and diversity of the RNL subfamily and a lack of data in Gymnosperms. We developed, searched and annotated transcriptomes assemblies of seven conifers and identified a resource of 3816 expressed NLR sequences. Our analyses encompassed sequences data spanning the major groups of land plants and determinations of NLR transcripts levels in response to drought in white spruce. We showed that conifers have among the most diverse and numerous RNLs in tested land plants. We report an evolutionary swap in the formation of RNLs, which emerged from the fusion of an RPW8 domain to a NB-ARC domain of CNL. We uncovered a quantitative relationship between RNLs and TNLs across all land plants investigated, with an average ratio of 1:10. The conifer RNL repertoire harbours four distinct groups, with two that differ from Angiosperms, one of which contained several upregulated sequences in response to drought while the majority of responsive NLRs are downregulated.

The NLR (nucleotide-binding, leucine-rich repeat) gene family is the main group of cytoplasmic receptors involved in the recognition of specific pathogens as part of effector-triggered immunity in plants ${ }^{1}$. NLRs may confer resistance to biotic aggressors including viruses, bacteria, fungi, nematodes and insects ${ }^{2-6}$, and abiotic stresses such as drought in some cases ${ }^{7}$, and present a wide range of variation across flowering plants ${ }^{8}$. This fundamental role of NLR proteins in resilience to biotic and abiotic stresses has encouraged genome-scale studies of their evolution, primarily in Angiosperms ${ }^{9-14}$.

The NLR protein family may have originated in green algae ${ }^{15}$ and was well-defined early in the land plant lineage ${ }^{16}$. It has evolved through gene duplication, domain fusions, and acquisition of amino-acid sequences that enable the detection of pathogens, protein dimerization, the activation of immune responses and possibly the triggering of a type of programmed cell death known as hypersensitive-like response ${ }^{17}$. The central nucleotide binding NB-ARC domain binds ADP or ATP molecules, which induces a protein conformation change and the switch from an 'OFF' to an 'ON' state. The NB-ARC domain, which is often used to perform phylogenetic studies, contains highly conserved motifs (Ploop, kinase, RNBS, GLPL, MHD) involved in intra- and extra-molecular

${ }^{1}$ INRA, Université Côte d'Azur, CNRS, ISA, 400 route des Chappes, BP167, 06903, Sophia Antipolis, France. ${ }^{2}$ Department of Plant Sciences, University of Oxford, South Parks Road, Oxford, OX1 3RB, UK. ${ }^{3}$ Forest Research Centre and Institute for Systems and Integrative Biology, Université Laval, 1030 rue de la Médecine, Québec, OC, G1V 0A6, Canada. ${ }^{4}$ Gydle Inc., 1135 Grande Allée Ovest Suite 220, Québec, QC, G1S 1E7, Canada. ${ }^{5}$ Center for Organismal Studies (COS), University of Heidelberg, Im Neuenheimer Feld 345, 69120, Heidelberg, Germany. ${ }^{6}$ Natural Resources Canada, Canadian Forest Service, Laurentian Forestry Centre, Québec, G1V 4C7, Canada. ${ }^{7}$ Département des Sciences Fondamentales, Université du Québec à Chicoutimi, 555 Boulevard de I'Université, Chicoutimi, OC, G7H2B1, Canada. ${ }^{8}$ Canada Research Chair in Forest Genomics, Université Laval, 1030 rue de la Médecine, Québec, QC, GIV 0A6, Canada. Correspondence and requests for materials should be addressed to C.V.G. (email: cyril.van-ghelder@inra.fr) 
interactions ${ }^{18,19}$. Among these motifs, the RNBS-A, RNBS-D and the MHD motifs show some variations that are correlated to the $\mathrm{N}$-terminal domain identity and can be used to classify the sequences into subfamilies. The C-terminal LRR domain mainly supervises the recognition function through interactions with effectors or molecules modified by effectors through residues in its horseshoe $3 \mathrm{D}$-structure ${ }^{20}$.

The conserved Toll interleukin-1 receptor (TIR) domain at the $\mathrm{N}$-terminus defines the large TIR-NB-ARC-LRR (TNLs) subfamily ${ }^{21}$, many members of which display a C-terminal post-LRR domain (PL) of unknown function ${ }^{22}$. The two other subfamilies are made up of non-TIR containing NLRs with a diversity of N-terminal sequences and structures: (1) the CC-NB-ARC-LRR (CNL) protein subfamily, which displays a coiled-coil structure in their N-terminus ${ }^{9}$ and (2) the RPW8-NB-ARC-LRR (RNL), which shares homology with RPW8-only proteins (RESISTANCE TO POWDERY MILDEW8) from Arabidopsis thaliana ${ }^{23}$. In addition, partial NLRs such as RBA1 (TIR-only protein), TN2 (TIR-NB-ARC-only), RPW8.1 and RPW8.2 (RPW8-only) were recently shown to confer resistance to pathogens ${ }^{23-25}$; therefore, we considered truncated versions in our study of the NLR gene family.

The occurrence of the different NLR subfamilies is variable among the major plant taxonomic groups. For instance, Monocots have many CNL encoding genes and lack the TNL subfamily ${ }^{21}$. The RNL subfamily is found in primitive plants such as Physcomitrella patens but has few or no members in other plant lineages, except in the Rosaceae, where it underwent a major expansion ${ }^{26}$. The RNL subfamily have been classified as a sister clade to the CNLs and TNLs ${ }^{11}$, but its evolution remains poorly resolved owing to the variability in both CNL and RNL subfamilies and its sequence motifs remain poorly defined. In Angiosperms, RNLs are subdivided into two identified subclades ${ }^{27}$ based on the homology of their RPW8 domain to the one of (1) the N-required gene 1 (NRG1), co-responsible of the Tobacco Mosaic Virus resistance in Nicotiana benthamiana ${ }^{28}$, or (2) the activated disease resistance gene 1 (ADR1), involved in both pathogen resistance and drought tolerance in Arabidopsis thaliana ${ }^{7}$. Functionally, RNL genes function as helper proteins acting downstream of sensor NLRs ${ }^{27,29-31}$.

The recent analysis of Pinus taeda L. and Picea abies (L.) H. Karst. genome drafts recently suggested the occurrence of numerous RNL sequences ${ }^{26}$ but a comprehensive analysis of conifer NLRs is lacking. A few conifer NLR sequences have been described ${ }^{32}$ and linked to resistance against pathogens such as Cronartium ribicola, which is responsible for the white pine blister rust ${ }^{33,34}$. Conifers possess very large genomes sizes and abundant pseudogenes, which hinder genome assembly and analysis of large gene families, but transcriptome sequencing provides an effective alternative to rapidly acquire large datasets of expressed genes ${ }^{35-37}$.

The overall goal of this study was to investigate the makeup of NLRs in conifers with the aim of improving our understanding of the RNL subfamily in plants. We introduced 3816 expressed NLR sequences in seven conifers in the Pinaceae and the Cupressaceae families. Our specific objectives were to: (1) identify the repertoire of expressed NLR sequences through the analysis of seven conifer transcriptomes; (2) classify the poorly known conifer NLRs based on amino acid domains and motif analyses; (3) investigate the diversity and the drought responsiveness of conifer RNLs, testing whether or not conifers form a single group external to the Angiosperm groups as recently proposed ${ }^{27}$. We found that conifer RNLs presented a level of sequence diversity that is unparalleled in tested Angiosperms and possibly in other plant taxa. Our results also suggest that they play a key role in drought response.

\section{Results}

NLRs are abundant and diverse in the Pinales. We developed a dataset of 3816 expressed NLR sequences (Supplementary Data S1) by analysing the transcriptomes of seven conifer species in five genera of the Pinaceae and Cupressaceae. The sequences were identified using the Pfam database (31.0) in the predicted amino acid sequence datasets obtained from the assembled mRNA sequence. The expressed sequences contained at least one of the canonical NB-ARC, RPW8, or TIR domains while CC-only and LRR-only were excluded as they may be mispredicted or not specific to NLRs. We filtered the full dataset to eliminate potentially redundant NLR peptides of a same encoding-gene. The number of expressed NLR genes thus identified ranged from 338 in Abies balsamea to 725 in Picea mariana (Table 1), in line with the total of 679 NLR genes identified in the genome of P. taed $a^{15}$. The total number of distinct NLR transcripts represented $0.73 \%$ to $1.35 \%$ of the assembled transcriptome depending on the species, which are substantial proportions of such genes when comparing with other Angiosperm transcriptomes (Supplementary Table S1).

Domain and motif specificities. The predicted NLR proteins followed the same domain organisation in conifers as described in Angiosperms, which included the differential N-terminal domains TIR, CC or RPW8, a conserved central NB-ARC domain, and a polymorphic C-terminal domain (LRR) (Supplementary Data S2). The TIR domain of conifer and Angiosperm TNLs was generally conserved, unlike the non-TIR N-terminus of the other NLRs, which was highly polymorphic and mainly made up of conformational domains (coiled-coil). The conifer TIR domain sequences were similar to those observed in Angiosperms and were characterised by a site displaying hydroxyl groups (poly-serine or threonine) close to the initial methionine, followed by the TIR-1 to TIR-5 motifs found in Angiosperms (Fig. 1). The TIR-1, 2 and 5 corresponded to the human TLR boxes 1 to 3 , respectively. Within the NB-ARC domain, highly conserved sequence signatures and variations were observed in conifers as found in Dicots. The conserved elements included the P-loop, Kinase 2, Kinase 3, RNBS-C and GLPL motifs, which are similar in all NLR subfamilies, and the RNBS-A, RNBS-D and MHD, which were specific to each of the NLR subfamilies (Fig. 1).

We report two specific signatures in the RNBS-D (CFLDLGxFP) and MHD (QHD) motifs that enabled an accurate detection of RNL sequences in conifer sequences. Among these motifs that have been previously identified in Angiosperms ${ }^{38}$, the RNBS-D motif was particularly useful to discriminate between different NLR sequences. It harboured conserved aspartic acid, glycine and cysteine residues that characterise each of the NLR forms (Fig. 1). We found that the QHD motif exclusively contained a glutamine (Q1) residue before the conserved 


\begin{tabular}{|c|c|c|c|c|c|c|c|c|c|}
\hline & & \begin{tabular}{|l|}
$\begin{array}{l}\text { Abies } \\
\text { balsamea }\end{array}$ \\
\end{tabular} & \begin{tabular}{|l|} 
Larix \\
laricina
\end{tabular} & \begin{tabular}{|l|}
$\begin{array}{l}\text { Picea } \\
\text { glauca }\end{array}$ \\
\end{tabular} & \begin{tabular}{|l|}
$\begin{array}{l}\text { Picea } \\
\text { mariana }\end{array}$ \\
\end{tabular} & \begin{tabular}{|l|} 
Pinus \\
banksiana
\end{tabular} & \begin{tabular}{|l|}
$\begin{array}{l}\text { Pinus } \\
\text { strobus }\end{array}$ \\
\end{tabular} & \begin{tabular}{|l|} 
Thuja \\
occidentalis
\end{tabular} & \begin{tabular}{|l|}
$\begin{array}{l}\text { Dist }^{\dagger} \\
(\%)\end{array}$ \\
\end{tabular} \\
\hline & Number of transcripts & 46178 & 50712 & 37491 & 58751 & 47473 & 45447 & 38767 & \\
\hline & TOTAL NLR & 338 & 633 & 506 & 725 & 486 & 560 & 486 & \\
\hline & Ratios & $0.73 \%$ & $1.25 \%$ & $1.35 \%$ & $1.23 \%$ & $1.02 \%$ & $1.23 \%$ & $1.25 \%$ & \\
\hline \multirow{12}{*}{ Non-TIR-NLR } & \multicolumn{9}{|l|}{ CNL } \\
\hline & $\mathrm{NB}_{\mathrm{CNL}^{-}}-(\mathrm{LRR})$ & 65 & 146 & 115 & 166 & 82 & 103 & 51 & \multirow{2}{*}{$22 \%$} \\
\hline & Ratios & $0.14 \%$ & $0.29 \%$ & $0.31 \%$ & $0.28 \%$ & $0.17 \%$ & $0.23 \%$ & $0.13 \%$ & \\
\hline & \multicolumn{9}{|l|}{ CNL2 } \\
\hline & $\mathrm{NB}_{\mathrm{CNL} 2}-$ (LRR) & 47 & 18 & 17 & 17 & 36 & 21 & 34 & \multirow{2}{*}{$6 \%$} \\
\hline & Ratios & $0.10 \%$ & $0.04 \%$ & $0.05 \%$ & $0.03 \%$ & $0.08 \%$ & $0.05 \%$ & $0.09 \%$ & \\
\hline & \multicolumn{9}{|l|}{ RNL } \\
\hline & RPW8 & 5 & 5 & 6 & 13 & 8 & 12 & 3 & \multirow{5}{*}{$9 \%$} \\
\hline & $\mathrm{NB}_{\mathrm{RNL}}-(\mathrm{LRR})$ & 11 & 8 & 6 & 10 & 21 & 12 & 13 & \\
\hline & RPW8-NB ${ }_{\mathrm{RNL}}-(\mathrm{LRR})$ & 16 & 33 & 19 & 16 & 26 & 16 & 15 & \\
\hline & Total RNL-related & 32 & 46 & 31 & 43 & 55 & 40 & 31 & \\
\hline & Ratios & $0.07 \%$ & $0.09 \%$ & $0.08 \%$ & $0.07 \%$ & $0.12 \%$ & $0.09 \%$ & $0.08 \%$ & \\
\hline \multirow{9}{*}{ TIR-NLR } & TNL & & & & & & & & \\
\hline & TIR & 43 & 100 & 121 & 144 & 94 & 89 & 72 & \multirow{6}{*}{$63 \%$} \\
\hline & $\mathrm{NB}_{\mathrm{TNL}^{-}}-(\mathrm{LRR})$ & 52 & 97 & 99 & 128 & 104 & 122 & 91 & \\
\hline & TIR-NB ${ }_{\mathrm{TNL}}-(\mathrm{LRR})$ & 55 & 143 & 58 & 105 & 52 & 126 & 154 & \\
\hline & TIR-LRR & 0 & 3 & 1 & 2 & 0 & 2 & 0 & \\
\hline & Total TNL-related & 150 & 343 & 279 & 379 & 250 & 339 & 317 & \\
\hline & Ratios & $0.32 \%$ & $0.68 \%$ & $0.74 \%$ & $0.65 \%$ & $0.53 \%$ & $0.75 \%$ & $0.82 \%$ & \\
\hline & Atypical NLR & 15 & 26 & 8 & 14 & 7 & 14 & 14 & \\
\hline & Undetermined NLR & 29 & 54 & 56 & 106 & 56 & 43 & 39 & \\
\hline
\end{tabular}

Table 1. Distribution of NLRs and their subfamily assignation in seven conifer transcriptomes. ${ }^{\dagger}$ The distribution is calculated using the total sequence number of each subfamilies in proportion of the total of assigned sequences (CNL, CNL2, RNL and TNL).

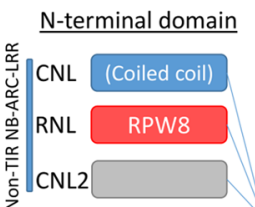

\section{Nucleotide Binding Domain (NB-ARC)}

\begin{tabular}{|c|c|c|c|c|c|c|}
\hline \multirow{2}{*}{$\frac{\text { P-loop }}{\text { (Kinase a) }}$} & \multicolumn{4}{|c|}{ RNBS-B } & & (Winged helix) \\
\hline & RNBS-A & Kinase-2 & Kinase 3 & RNBS-C & GLPL & $\begin{array}{ll}\text { RNBS-D } & \text { MHD }\end{array}$ \\
\hline
\end{tabular}
KNBS-A Kinase-2 Kinase
Leucine Rich Repeat $\underline{(L R R)}$

Pfam:

LRR1, LRR4, LRR5, or LRR8 Superfamilies: Ldomain or RN

TIR

$\begin{array}{lllllll} & T N & 1 & 2 & 3 & 4 & 5\end{array}$

TIR-1: YDVFINHRGPD

TIR-2: GLRVFLDXXELQXGD

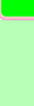

TIR-3: ASVHVAIFSxXYAXSXWCLXEL

$\cong$ TIR-4: IIPVFYXVXP

TIR-5: KWRXALS

$1 \ldots 5 \ldots 10.1 \ldots 5 \ldots 10.1 \ldots 5 \ldots 1 \ldots 5 \ldots 1 \ldots 5 \ldots 10 \ldots 15 \ldots 20 \quad 1 \ldots 5 \ldots$

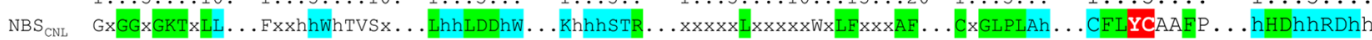

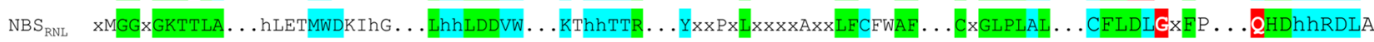

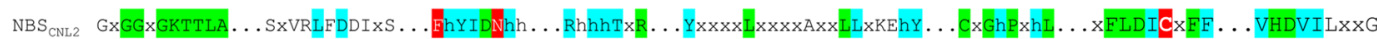

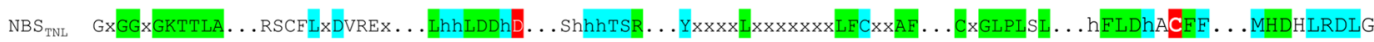

Figure 1. Schematic representation of the four subfamilies of NLRs in conifers. The detailed motifs of the NB-ARC domain are split into conserved motifs through NLR subfamilies (green) and discriminative motifs (grey). Pfam and Superfamilies entry identifiers are indicated above the green bar. Discriminant amino acids are highlighted in red, 'h' indicates various hydrophobic residue.

histidine (H2) and aspartic acid (D3) in the conifer RNL sequences, in contrast to the conventional methionine (M1) or other hydrophobic residues found in CNL and TNL proteins. We also identified an unconventional non-TIR NLR subfamily (CNL2), which had a unique combination of features in the NB-ARC domain, including a conserved cysteine in the sixth position (C6) after the conserved aspartic acid (D4) in the RNBS-D motif, a VHD motif and a polymorphic RNBS-A motif (Table 1, Fig. 1). A blastp analysis based on the RNBS-D motif only identified sporadic NB-ARC $\mathrm{CNL}_{2}$ sequences in recent Angiosperms. 
We scanned the conifer sequences for conserved C-terminal signatures downstream of the LRR domain but none were clearly identified, in contrast to Dicot TNLs where post-LRR (PL) domains are ubiquitous. None of the known Dicot PL motifs were detected in the entire dataset of predicted peptides by using blastp or FIMO analysis. We also searched in datasets from ancient plants among Embryophytes (P. patens, Sphagnum fallax), Ginkgo biloba, another conifer (P. abies), and an early-diverged Angiosperm (Amborella trichopoda), and only found complete PL motifs in A. trichopoda and a few partial ones in G. biloba. These observations suggest a partial acquisition early in seed plant evolution, and a potential adaptive expansion in early flowering plants (Supplementary Fig. S1).

Conifer sequences are broadly represented in all NLR subfamilies. We assigned each of the transcripts to specific subgroups CNL, CNL2, RNL or TNL based on N-terminal sequences and specific motifs in the NB-ARC domain when possible, considering only the sequences containing bona fide NB-ARC domains. The TNL sequences were most abundant in all of the species and represented $63 \%$ of the total (Table 1), except in $A$. balsamea, which harboured as many CNLs as TNLs. The respective proportions of the different subfamilies were similar in the two Picea species and Larix laricina $\left(\chi^{2}, P>0.999\right)$, the proportion of TNL sequences was slightly higher in Thuja occidentalis and Pinus strobus and lower in A. balsamea, while RNL sequences were most abundant in Pinus banksiana ( $0.12 \%$ of all the transcripts) (Table 1$)$.

Retracing the origin and distribution of RNL identifies a potential link to TNL evolution. The analysis of NB-ARC motifs is crucial to assign sequences to particular subfamilies. We reliably identified

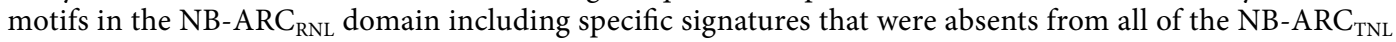
or $\mathrm{NB}-\mathrm{ARC} \mathrm{CNL}_{\mathrm{CN}}$ sequences, owing to the large and relatively uniform numbers of RNL sequences in conifers (Table 1). We used these results on RNL motifs to compare conifers and ancient plants to gain insights into the formation of RNL, and to investigate RNL sequence contingent sizes across land plants, aiming to explain differences observed in the seed plant phylogeny.

In early land plants, we identified two, four and ten putative RNL sequences in Marchantia polymorpha, $P$. patens and S. fallax, respectively, by using RPW8 and NB-ARC signatures. Although we had detected RPW8

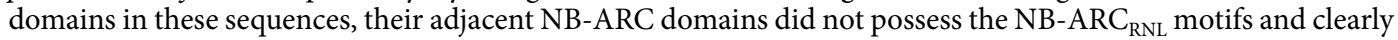
displayed CNL signatures in the RNBS-A, RNBS-D and MHD motifs (Supplementary Fig. S2). Among all the plant proteomes tested, this peculiarity was only found in the early land plants. These same species also harboured an RPW8-containaning sequence associated with other domains such as Ubox/Arm, PLAC8 and PKinase (Mapoly0115s0019.1.p, Pp3c12_21520V3.1.p, Sphfalx0341s0009.2.p). The data showed that the RPW8 domain fused to a core $\mathrm{NB}-\mathrm{ARC}_{\mathrm{CNL}}$ or connected to other domains already existing in early land plants.

The abundance of RNLs was then investigated in 42 plant proteomes distributed among diverse taxonomic groups of land plants in addition to those of the seven conifers (Fig. 2 and Supplementary Table S2). Zhong and Cheng (2016) identified the expansive RNL subfamily in several of the Rosaceae using blastp and also suggested that the Pinus and Picea had numerous RNLs ${ }^{26}$. Using the same blastp procedure, we also found large RNL contingents in both Pinus and Picea as well as in the two other groups of the Pinaceae family, namely Abietoideae and Laricoideae, and in the Cupressaceae family (Fig. 2a). We also detected thirteen RNLs in the early-diverged Gymnosperm G. biloba (Fig. 2a and Supplementary Table S2), suggesting that significant RNL contingents may be a common feature of Gymnosperms.

The data showed large variations across the land plants ranging from a single RNL sequence in Monocots to more than 30 in some of the Angiosperms without a clear pattern related to phylogeny. We retrieved TNL sequences from the same 42 plant proteomes either from prior surveys of NLR sequences or by using blastp searches (Fig. 2a and Supplementary Table S2) and performed a regression analysis between the numbers of RNLs and TNLs among the major groups of land plants. A significant relationship was uncovered, showing a 1:10 ratio (RNL:TNL; Fig. 2b). The overall regression model was significant, $F(1,47)=130.36, P=3.75 \mathrm{e}-15, \mathrm{R}^{2}=0.73$ (Fig. 2b). A similar analysis between RNLs and CNLs failed to detect any quantitative relationship (Fig. 2c).

Conifers present a high diversity in RNL gene sequences. We tested whether the large number of conifer RNLs was associated with increased sequence diversity, which could represent an ancient sub-group expansion. We extracted and trimmed 185 full-length RPW8 domains from the seven conifer sequences to identify potential subgroups. We only retrieved RPW8 domains from RPW8-only and RNL peptides. Following the approach described by Zhong \& Cheng $(2016)^{26}$, we produced an unrooted tree including all of the full-length conifer RPW8 domains and four Angiosperm reference RPW8 domains to delineate the diversity of conifer RPW8 and assign sequences to specific groups. The resulting tree contained four distinct groups of unequal size (Fig. 3a). Two of the groups clustered with the Angiosperm sequences ADR1 (Group 2) or with the weakly supported NRG1 group (Group 4). The tree contained two other large groups (1 and 3) entirely made up of conifer sequences (Fig. 3a). Each of the seven species investigated here appeared to be homogenous in diversity among the four conifer groups. Considering the group 1, the Thuja sequences were entirely contained in subgroup 1b, suggesting an ancient divergence followed by recent duplications specific to this group of sequences (1) in the Cupressaceae (Fig. 3a). The group 2 represented by ADR1 had the most uniform representation, with three sequences on average per conifer species (ranging from two to five in T. occidentalis and P. banksiana, respectively).

Each of the groups was submitted to a clustering filter, which showed strong sequence clustering in groups 1 and 3 using a cut-off value of $80 \%$ identity, further suggesting multiple duplications for some of the sequences (Supplementary Fig. S3). The tree topology obtained with the representative sequences by using an identity-based clustering (cut-off of 60\%) did not differ from the groups delimited with the full set of sequences (Supplementary Fig. S4). We carried out a motif analysis of the RPW8 domain in the four groups separately and found increasing 


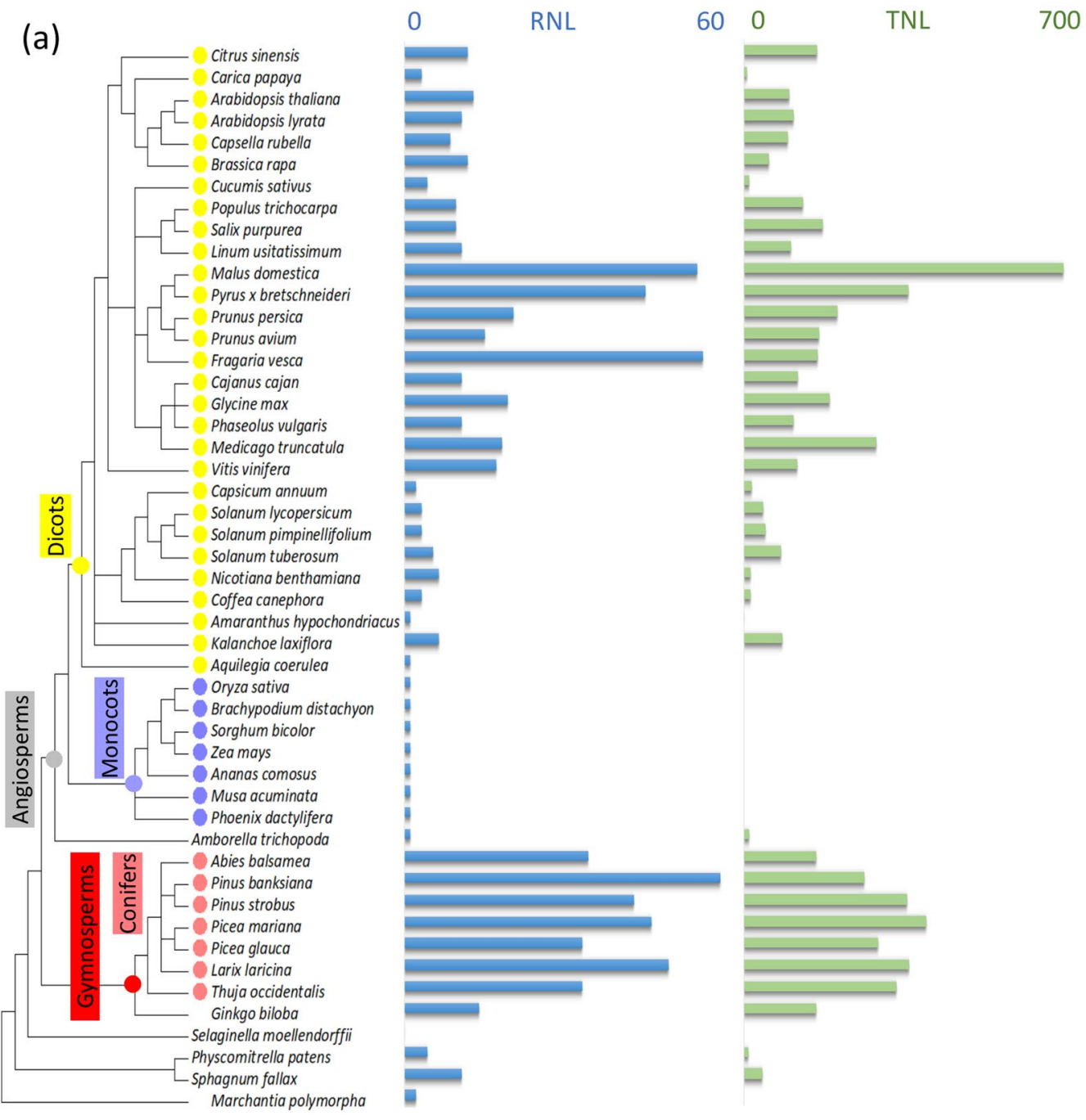

(b)

(c)
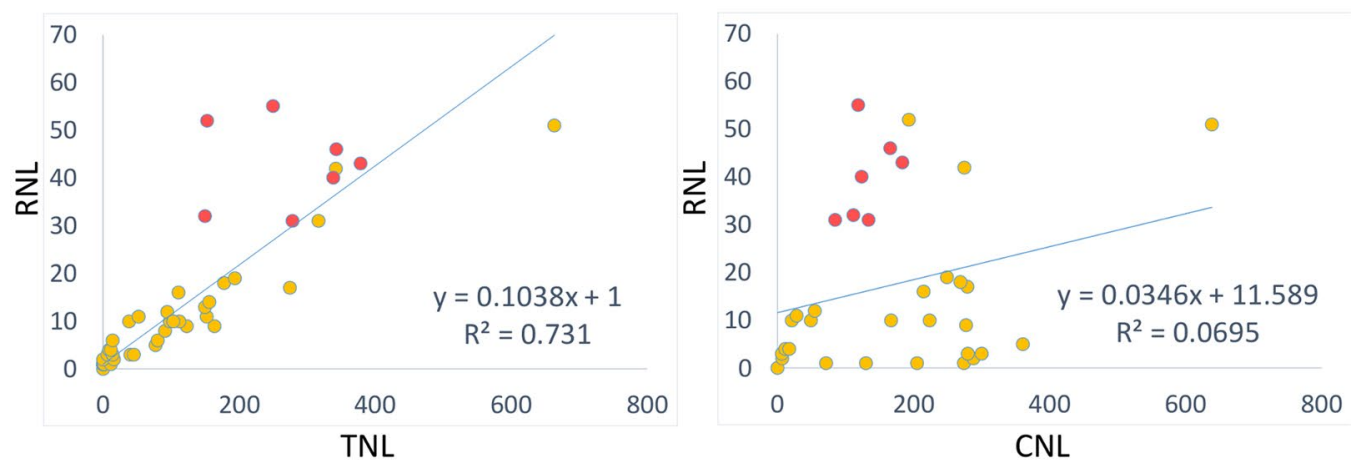

Figure 2. Distribution of the RNL and TNL genes in 49 land plants. (a) RNL- and TNL-related sequence numbers are mapped to the species tree created from the NCBI Common Taxonomy Tree (https://www.ncbi. nlm.nih.gov/Taxonomy/CommonTree/wwwcmt.cgi). Abscissa axis represents the protein numbers situated above the diagram. (b) RNL versus TNL numbers. (c) RNL versus CNL numbers. Light red dots represent the conifer species used in this study. The linear regression equation and the $\mathrm{R}^{2}$ coefficient are indicated.

polymorphism in the $\mathrm{N}$ and C-termini from groups 1 to 3 and a conserved central section in the RPW8 domain (Fig. 3b and supplementary Table S3). Some motifs show signs of recombination (f), loss (a, e) or de novo formation (b, h, i). The group 4 appeared to be more diverged compared to groups 1 to 3 (Fig. 3b). 


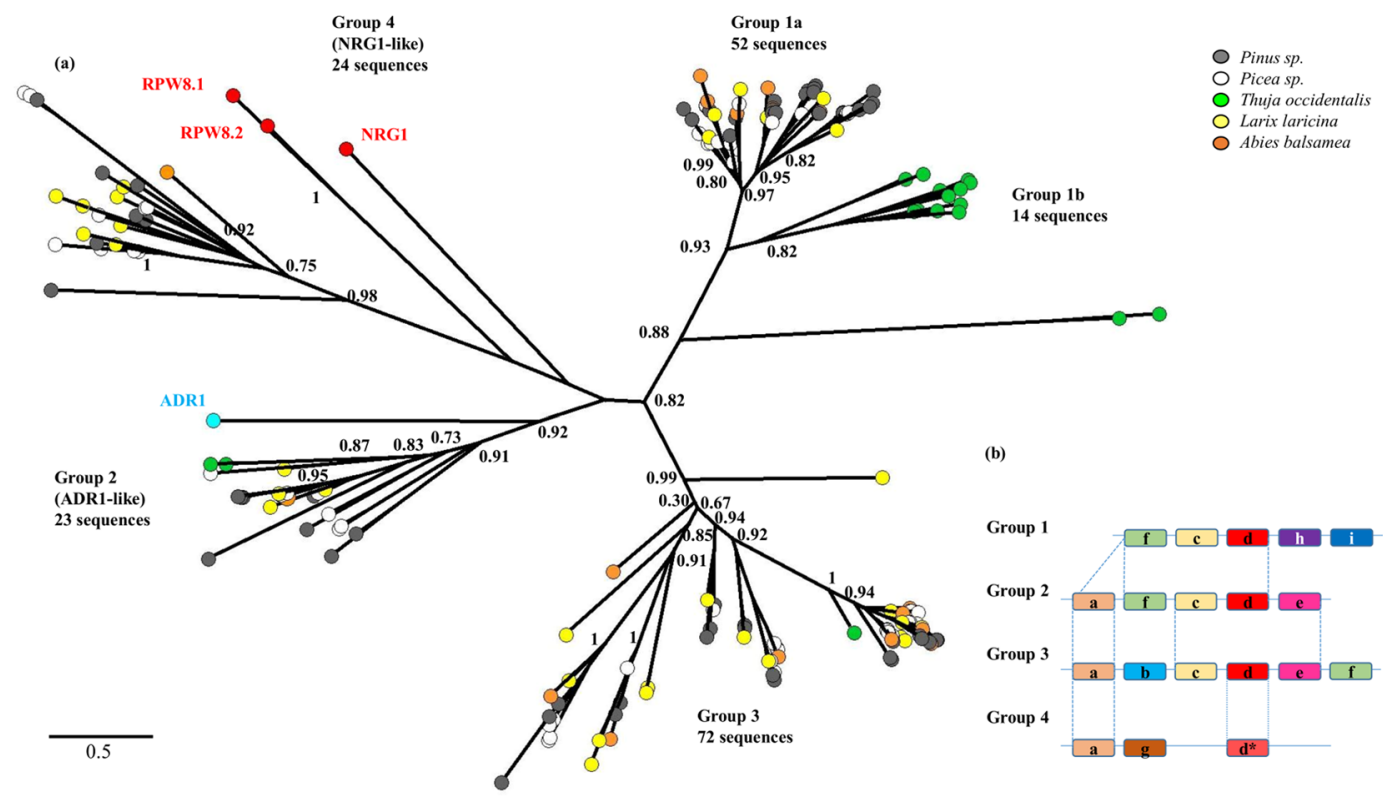

Figure 3. Unrooted ML phylogenetic tree and motif analysis for the RPW8 domain. (a) 185 RPW8 domains identified in conifers in this study together with RPW8 domains of ADR1 (blue dot), RPW8.1, RPW8.2 and NRG1 (red dots) were selected to build the ML phylogenetic tree. The number of conifer sequences contained in each of the groups are indicated below group numbers. For clarity, only main support values $>0.70$ are displayed (full data available in Supplementary Fig. S6). (b) Motif pattern diversification within the RPW8 domain associated with each group. The details of the motifs are available in Supplementary Table S3.

Next, we examined the conifer diversity of RNLs in view of the RNL land plant sequences. We retrieved the RPW8 domain from other plants (counted sequences in Fig. 2), together with Picea abies peptide sequences obtained from an independent dataset ${ }^{39}$, and applied an identity-based clustering (cut-off of $60 \%$ ) on all of the sequences to examine the extent of RPW8 domain diversity. We thus obtained a final set of 197 RPW8 sequences to build an expanded unrooted tree. The RNL sequences generally clustered according to their taxonomic clades within each of the groups (Fig. 4). The Gymnosperm sequences P. abies and G. biloba clustered together with the conifer sequences reported in this study (groups 1-4). All of the Angiosperm sequences clustered into groups 2 and 4 (Fig. 4). All the tested Monocot sequences only clustered in the ADR1 group (2). Most of the sequences of the early land plants, presumably the ancestors of RNLs, were located in NRG1 group (4). A few sequences of $S$. fallax also clustered in the Gymnosperm group 3, indicating a probable onset of RNL diversification. The phylogenetic tree also highlighted some particular sequences that underwent large expansions in conifers (group 3) or Rosaceae (group 4) (Fig. 4).

NLRs are differentially expressed under drought conditions. We investigated the drought responsiveness of NLR genes by RNA-seq analysis of samples from an independent study in P. glauca by Stival Sena et $a l .{ }^{40}$. The study monitored plants of three unrelated genotypes that were well-watered (controls) or not watered at all (drought treatment) over a 22-day period, with sampling time points at $0,7,14,18,22$ days. A principal component analysis of the entire dataset of foliage transcript levels showed that the drought treatment was the main factor that explained $53 \%$ of the observed variance (Supplementary Fig. S5) and supported samples groupings by treatment and genotype. The data showed major effects of the drought treatment after 18 and 22 days, independently of the clone used, in addition to smaller and more variable effects between clones at 14 days (Supplementary Fig. S5).

We identified 119 differentially expressed (DE) NLR genes (adjusted p-value $<0.05$ ) in drought-treated plants compared to well-watered controls across the NLR repertoire, independently of the genotype. The major changes in NLR expression were observed after 14 days of treatment and increased at 18 and 22 days, thus following the physiological trends in water potential (Fig. 5). The data showed that a large majority ( $>87 \%$ ) of the DE NLR genes were downregulated, from slightly (the TNL, PG_019785_T.1; Log2FC $=-0.25$ ) to strongly downregulated (the CNL, PG_022220_T.1; Log2FC < -5) (Fig. 5b and Supplementary Table S4). We found that all NLR subfamilies were represented in the pool of DE sequences. A clustering analysis based on expression patterns identified 14 groups including six expression groups that only contained members of one subfamily (Fig. 5 and Supplementary Table S4).

While the DE CNL and TNL sequences were almost exclusively downregulated, $62 \%$ of the RNLs were upregulated. In total, 8 out of all the 15 upregulated NLRs belonged to the RNL subfamily (Fig. 5).

Gymnosperm-specific RNLs have drought responsive expression. We identified 13 drought responsive RNL genes, including 8 upregulated (up to $\log 2 \mathrm{FC}>8$ ) and 5 downregulated sequences (up to 


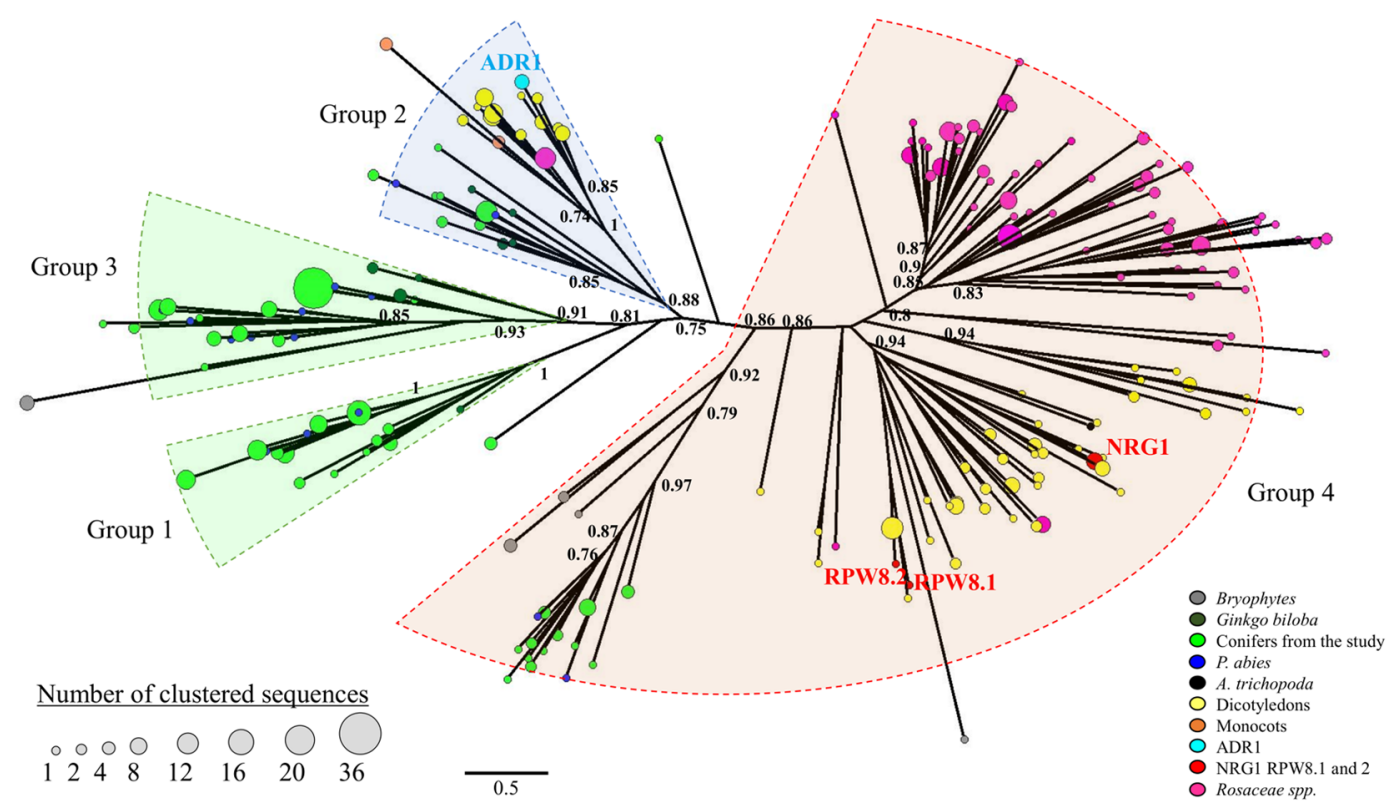

Figure 4. Unrooted ML phylogenetic tree of the RPW8 domain in land plants. 197 representative RPW8 domains retrieved from 50 land plants ( 49 species used in the Fig. 3 and Picea abies) were used for the phylogenetic analysis. The size of the circles reflects the number of sequences that are clustered (sequence identity cut-off of 0.60 ) with the representative sequence. For clarity, only main support values $>0.70$ are displayed (full data available in Supplementary Fig. S7). Colours are used to reveal taxonomic groups and reference sequences.

a)

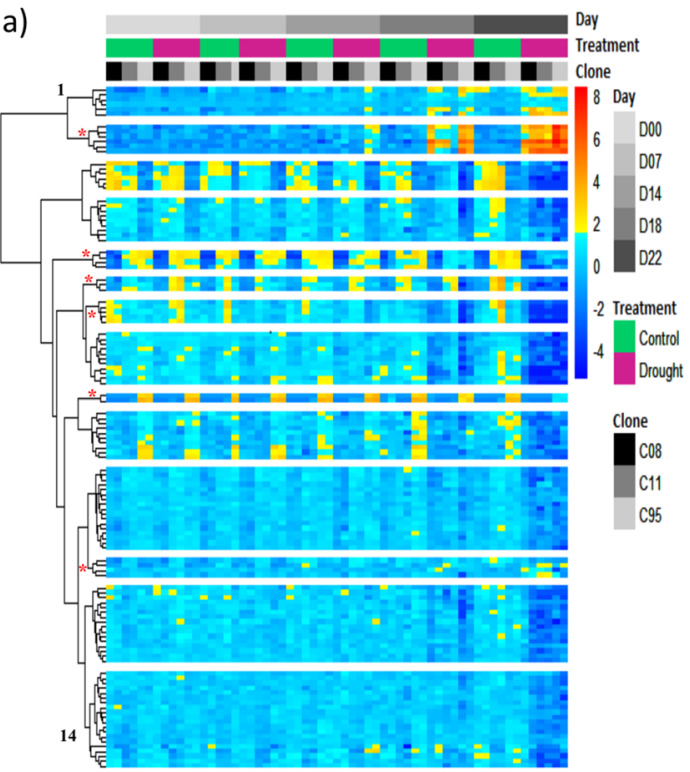

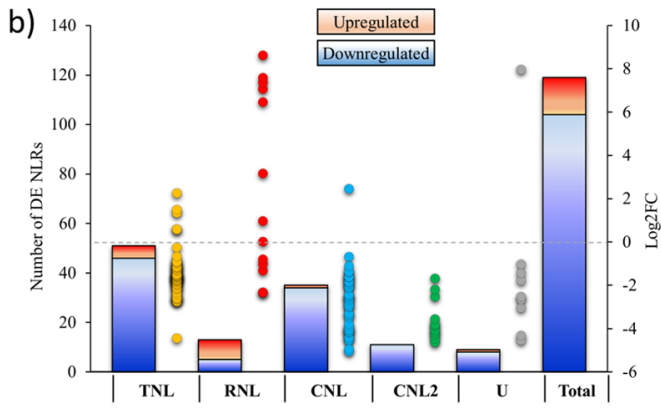

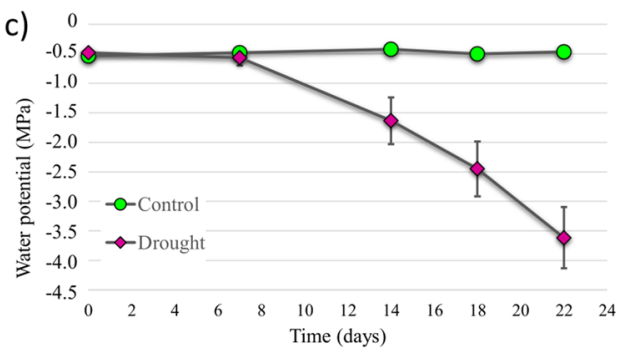

Figure 5. Expression patterns and distribution of drought responsive NLR genes in Picea glauca. (a) Heatmap illustrating the 14 expression groups formed of the 119 NLR sequences with drought responsive transcript levels for each genotype, treatment and sampling day. Red asterisks indicate expression groups with a single subfamily of NLR. (b) Number of drought responsive NLR genes and Log2 Fold Change observed in the four subfamilies and undetermined $(\mathrm{U})$. (c) Measurement of water potential in needles for control and water-stressed plants at five sampling times. Each point is the average of 12 biological replicates ( 3 P. glauca genotypes with 4 replicates each) with standard deviation (adapted from Stival Sena et al. ${ }^{40}$ ).

Log2FC <-2) (Fig. 6 and Supplementary Figure S9). Examining the set of five most upregulated RNL sequences, we observed a steady increase in transcript levels starting from the day 7 , which was before any noticeable change was observed in the leaf water potential (Fig. 5). All of the 13 drought responsive RNLs belonged to the group 1 


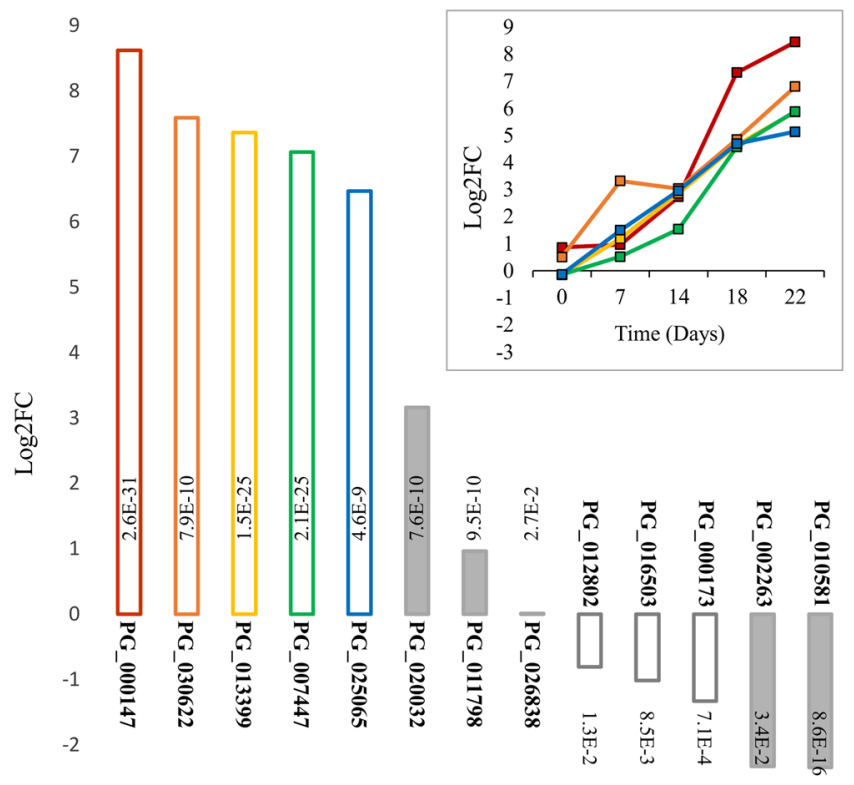

RNL Group 1 aRNL Group 3

Figure 6. Expression of the 13 drought responsive RNLs and their RNL group assignation. The main figure shows transcript levels (Log2 fold change) after 22 days without water. Adjusted p-values are the result of the statistical tests for differential expression between drought-treated and control groups. Time course of expression profiles (Log2 fold change) are shown for the five most upregulated RNLs (top).

or 3 of sequences that are specific to Gymnosperms. None of them clustered with the ADR1 or the NRG1 group of sequences (group 2 and 4) that are in common with Angiosperms. This observation suggests that group 1 and 3 likely harbour gene sequences specifically involved in the response to drought in conifers (Fig. 6).

A genomic cluster of group 1 RNL sequences was identified based on the P. glauca linkage map ${ }^{41}$. The sequences were identified based on similarity (99\% identity) between the full set of $P$. glauca RNL sequences described here and the sequences that were positioned on the linkage map ${ }^{41}$. Many of the RNL sequences did not produce any matches but four RNL sequences with matches were localised within 0.4 centi Morgans (cM) of each other (positions 3.86 to 4.18) on linkage group 6. Their patterns of expression ranged from downregulated (PG_010581_T.1), upregulated (PG_011798_T.1) to not differentially expressed genes (PG_012538_T.1; PG_008655_T.1) (Supplementary Table S5).

\section{Discussion}

Most NLRs are constitutively expressed in healthy tissues and some are stress responsive in plants ${ }^{20}$. We produced a transcriptome-wide repertoire of NLR sequences from seven species in the Pinaceae and Cupressaceae families by RNA sequencing of several tissues and species in normal and stressed conditions. We found a large and varied NLR gene arsenal in these conifers compared to Angiosperms that have reduced or even eliminated either TNL or RNL subfamilies. We discuss interspecific variations and evolution in non-TIR NLRs including their ratio to TNLs, which may depend on their involvement in successful stress responses, resulting in their selection followed by expansion through duplications in some cases.

Motif usage in conifers and implications in NLR evolution. Extensive studies on NB-ARC domains have highlighted their motif flexibility involving the reorganisation of pre-existing building blocks that can be observed at a domain or motif level ${ }^{16}$. In our dataset, we observed conserved motifs (P-loop, Kinase $2 / 3$, and GLPL) involved in crucial ADP/ATP binding and shifting ${ }^{42}$. These motifs are conserved in all NLR subfamilies and in NB-ARC of prokaryotes, which do not display upstream N-terminal domains ${ }^{16}$. Thus, these motifs are not influenced by the $\mathrm{N}$-terminal domain identity. The over-representation of RNL sequences in several conifers allowed us to define reliable $\mathrm{NB}-\mathrm{ARC}_{\mathrm{RNL}}$ signatures. We showed that RNL proteins strictly required a glutamine, first residue of the MHD motif in Fig. 1 (Q1) in place of the methionine (M1) (i.e. QHD). This glutamine residue was first identified in Arabidopsis ADR1 homologs ${ }^{43}$ and probably possesses a functional role through its ability to create hydrogen bonds possibly involved in ATP processing. Indeed, mutations on $\mathrm{H} 2$ or D3 residues in the MHD motif (Fig. 1) favour ATP binding leading to the auto-activation of NLR and cell death ${ }^{44,45}$. The shift in sensitivity between the ON/OFF states is crucial to confer a finite lifetime to active forms and reliably unlock OFF states initiating safe cell death ${ }^{46}$. The RNBS-D motif is shaped according to the N-terminal domain identity but its exact function remains unclear ${ }^{19}$. By contrast to other NLR subfamilies, RNLs did not harbour a conserved cysteine (C5, C6 or C7) but a conserved glycine in the sixth position (G6) of the RNBS-D motif (Fig. 1). The ability of cysteine residues to form disulphide bonds may play a role in intramolecular interactions whereas the glycine in RNLs could reduce the steric hindrance. Our motif analysis showed that CNL2 sequences display polymorphic 
motifs and an intermediate RNBS-D form that resembles that of the archaic kinase-NB-ARC-LRR in P. patens ${ }^{10}$. These findings and the near absence of CNL2 sequences in plants other than conifers suggest that CNL2 could represent an intermediate form in the evolutionary swaps between NLR subfamilies.

Diversity and drought responsiveness of RNL genes. The data reported here showed that conifer RNLs include sequences that are distinct from those previously described in addition to members of the well-known ADR1 and NRG1 groups ${ }^{26,27}$. The presence of ADR1-like sequences among all flowering plants and the absence of NRG1-like representatives in some taxonomic groups reinforced the previously confused state of knowledge $\mathrm{e}^{27,43}$. We showed that conifer RNL genes split into four groups, two of which correspond to the only known Angiosperm groups (groups 2 and 4) while the two others (groups 1 and 3) are Gymnosperm-specific (Figa 4,5). Our results are at odds with the suggestion of a single independent Gymnosperm group ${ }^{27}$ and places the NRG1-ADR1 differentiation before the divergence between Angiosperms and Gymnosperms, at more than $300 \mathrm{Myr}$ ago ${ }^{47}$. RNL sequences from G. biloba and P. abies support this interpretation (Fig. 5).

Although they are usually found in low numbers, we detect that ADR1-like sequence group has a few sequences in all of the seed plants tested to date, which contrast with other RNL groups. Taken toghether, the absence of plant species lacking ADR1-like sequences, the involvement of ADR1 in Arabidopsis thaliana drought tolerance and its identification as a cornerstone in NLR signalling ${ }^{31}$ clearly suggest that ADR1-like sequences are crucial for plant survival and fitness. Considering the phylogenetic distance between species, the compact aspect of the corresponding branch in the phylogenetic tree supports a significant sequence conservation previously documented in flowering plants ${ }^{43}$. It has been suggested that ADR1-like proteins carry out a general function in disease resistance or play a critical role to control common pathogen ${ }^{27}$. Its characteristics may also fit with a role in adaptation to abiotic stresses that affect most plants in their natural habitats, or a hub function acting downstream of a sensing network. In contrast, the heterogeneous NRG1-like group is made up of more polymorphic sequences, which is reflected by its scattered distribution in the phylogenetic tree (Fig. 4).

Most importantly, our data identify two Gymnosperm-specific groups (1 and 3) of RNLs that may harbour many recent duplications based on their lower levels of sequence divergence. The RPW8 domain motif organisation in these latter two groups shows relatedness with ADR1-like sequences, suggesting a shared origin with the ADR1 group. The extensive duplication and diversification of RNLs in conifers argues in favour of a role in adaptation to ubiquitous stress such as harsh environmental conditions.

Our RNA-Seq analysis in P. glauca suggests that the majority of differentially expressed NLRs are downregulated after a water deprivation of several days, which is in line with increased susceptibility to pathogens in stressed conifers $^{48,49}$. Interestingly, only RNLs from groups 1 and 3 appeared to be responsive to drought. Specifically, a subset of RNLs from group 3 were strongly overexpressed after drought treatment and few sequences were slightly downregulated, as observed for a majority of stress-responsive NLRs. In Arabidopsis, ADR1 was shown to be actively involved in drought tolerance ${ }^{7}$. Here we report for the first time in conifers, RNL sequences from a nearby group of ADR1 that respond significantly and early to drought stress. Their genomic localisation indicates that some of them may be clustered in tandem arrays. The comparative study of drought-responsive RNL clusters among clones or species that show differential resilience to drought should be of interest. Analyses of copy number variation, gene organisation, sequence diversity or expression among these clusters may reveal molecular mechanisms acting in adaptation to drought in conifers ${ }^{50}$.

RNL origin and distribution in plant lineage. The recent sequencing of ancient genomes has shed light on the origin of NLR proteins ${ }^{15}$. The RNL subfamily arose before the Gymnosperm - Angiosperm split ${ }^{27}$ putatively de novo from a non-coding region of an NLR-encoding gene or following domain fusion ${ }^{26}$. No RNLs are mentioned in green algae ${ }^{15}$; nevertheless, we identified a few RNL sequences in the tested early land plants, including the liverwort M. polymorpha, and in the mosses $P$. patens and $S$. fallax, which harboured typical NB-ARC ${ }_{\mathrm{CNL}}$ but no $\mathrm{NB}-\mathrm{ARC}_{\mathrm{RNL}}$ motifs. In these three species we also identified other RPW8-containing proteins. NB-ARC $\mathrm{RNL}_{2}$ signatures were detected in all tested seed plants, including ancient plants such as G. biloba and A. trichopoda. It is possible that a pre-RNL emerged from a pool of CNL sequences in which the fusion of the RPW8-encoding exon occurred early in land plant followed by a transition toward RNL forms that arise before the divergence between Gymnosperms and Angiosperms. However, a study aiming to confirm this hypothesis and to decipher the RNL evolutionary history should include more sequences from ancient plant lineages (e.g. green algae, mosses, lycophytes, and ferns) but also other Gymnosperm sequences from Cycads and Gnetophytes. Expansion of the RNL sequence family is observed in groups 1 and 3 in the conifers and in group 4 in the Rosids. By contrast, taxonomic groups such as the Monocots have only a single ADR1-like RNL and have lost any other group representatives. A striking co-absence of NRG1-like and TNL sequences has been highlighted in Lamiales (a subgroup of Asterids), early-diverged Dicots (Aquilegia coerulea), and Monocots ${ }^{27}$.

We identified a high abundance of both RNL and TNL genes in conifers. We also observed that the number of TNL genes correlates well with that of RNLs but not with that of CNLs in diverse seed plants. The quantitative link between TNLs and RNLs (approximate ratio of 1:10, RNLs:TNLs) is based on 49 plant species, despite potential variations in determinations of gene numbers among species. This asymmetrical ratio linking the abundances of RNLs and TNLs is surprising especially given that the two subfamilies are probably not physically linked, e.g. the chromosome 7 of Prunus persica contains $80 \%$ of the RNL pool but only $5 \%$ of the TNLs (Supplementary Table S6), and the dynamic NLR family has undergone several gene expansions and losses during evolution ${ }^{11}$. In Angiosperms, the functionally characterised RNLs NRG1 and ADR1 act as downstream helpers of conventional sensor NLRs ${ }^{29,51}$. NRG1 acts downstream of the activation of the TNLs N, Roq1 and RPP1, but not the CNLs Bs2 and Rps $2^{28,30}$. ADR1 and its paralogues are also thought to act in conjunction with TNL and CNL sensors ${ }^{29,31}$. Recently, an extensive study revealed a larger NLR network involving numerous TNLs, including pair TNL pairs, and a few CNLs that signal via NRG1 and/or ADR1 ${ }^{31}$. In this context, the proportionality between TNLs and 
RNLs may be explained by the structure of their functional relationship (i.e. multiple sensors that signal via few helpers). RPW8 domains recruit TNL gene-signalling components such as EDS1, PAD4 and EDS5, but do not recruit CNL gene-signalling components ${ }^{52}$. New findings showed that signalling machineries, including EDS1, PAD4 and SAG101, co-evolved within plant species and clades for regulating downstream pathways in TNL immunity ${ }^{53}$. A recent study that highlighted the loss of numerous NLRs and their gene-signalling components in aquatic Angiosperms paves the way to decipher the complex interactions between the plant immune system and drought tolerance ${ }^{54}$. All together, these results support the hypothesis that RNLs may function redundantly in network, together with TNLs to activate NLR-triggered immunity. By contrast to the complex network recently identified in Solanaceae that is exclusively composed of sensor-helper CNLs ${ }^{55}$, the complex TNLs/EDS1/RNLs might have evolved independently to provide a parallel machinery that triggers immunity especially in Rosids and in Gymnosperms.

\section{Methods}

Plant material and RNA extraction for transcriptomes. The study relied on four year-old saplings and germinated seeds of seven conifer species, including two spruces (Picea glauca and Picea mariana), two pines (Pinus strobus and Pinus banksiana), a fir (Abies balsamea), a larch (Larix laricina) of the Pinaceae family and a representative of the Cupressasseae family (Thuja occidentalis). All of the individuals were obtained from open-pollinated seed lots. The saplings were cultivated under standard greenhouse conditions ${ }^{56}$ and included four individuals per species. Before the sampling, we deprived half of the saplings of each species of water for 14 days and watered the other half normally. We sampled six vegetative tissues from these greenhouse grown plants. Total RNAs were extracted from the different tissues by using the protocol described in ${ }^{57}$ with modifications ${ }^{58}$, and the MasterPure $^{\mathrm{TM}}$ Plant RNA Purification kit. The detailed protocol is available in Supplementary Methods.

RNA-Seq library synthesis, sequencing and assembly. We sampled different conifer tissues and applied a drought stress to generate a maximum number of RNAs. We pooled all of the RNAs from the different tissues for each species separately (Supplementary Methods). We used $500 \mathrm{ng}$ of total RNA from each of the species pools to synthetize mRNA libraries using TruSeq ${ }^{\circledR}$ Stranded mRNA kit following the supplier's instructions. Second strand synthesis and post PCR clean-up were performed using Axygen ${ }^{\circledR}$ AxyPrep ${ }^{\mathrm{TM}}$ Mag PCR Clean-Up Kit. Each library was quantified using a Nanodrop ND-1000 and characterised with an Agilent Bioanalyzer 2100 using High Sensitivity DNA chips. The Genome Quebec Innovation Centre at McGill University (Montreal, Quebec, Canada) carried out the sequencing of each pool using the "rapid run" procedure (paired-end $250 \mathrm{bp}$ ) with an Illumina HiSeq 2500 sequencing system.

The transcriptome assemblies were performed with Gydle software NUCLEAR version 3.2.16, RESOLVE version 2.6.12 and VISION version 2.6.12, and guided using the white spruce gene catalogue obtained by the sequencing of full-length insert cDNAs (FLICs) from white spruce cDNA libraries as described in ${ }^{59}$. The detailed protocol of assembly is available in Supplementary Methods. The accessions numbers for all of the transcriptome assemblies and the sequence reads are mentioned in the additional data and a detailed list is available in Table 2 of the Supplementary Methods.

Functional annotation and motif discovery. For each of the seven conifer species, we applied a first filter by analysing the entire predicted protein dataset using the Pfam database with a cut-off value of 1.0. Sequences displaying the Pfam entries NB-ARC (PF00931), TIR (PF01582), RPW8 (PF05659) and LRR 1 to 6, 8, 9, and LRRNT (PF00560, PF07723, PF07725, PF12799, PF13306, PF13516, PF13855, PF14580 and PF01462, respectively) were extracted. Then, we characterised the domain organisation using HMMER web server version 2.19.0 with the selection of Pfam, TIGRFAM and superfamily HMM databases with significance e-values set to 0.01 (model) and 0.03 (Hit) ${ }^{60}$. Atypical NLR sequences were also verified with InterProScan by using the entire database available. Motifs analysis and scanning were carried out using the MEME suite ${ }^{61}$ with maximum number of motifs set to 10, minimum-maximum motif width set to 5-30 and 5-50 and minimum sites per motif equal to 2 .

Additional data sampling and extraction data. The full list of additional plant protein datasets and the versions that were screened is available in Supplementary Methods. RNL sequences were extracted using BlastP searches with (i) RPW8 domains of ADR1 (BAF00531.1), NRG1 (AAY54606.1), RPW8.1 (ACJ05907.1), RPW8.2 (ACJ72031.1) and a representative of each of the four conifer RNL clades detected in this study and (ii) NB-ARC $\mathrm{RNL}_{\mathrm{L}}$ laying between the RNBS-D and the QHD motifs as queries with broad e-value $<1$. When TNL numbers were not available in the literature, we produced our own estimates by using BlastP (e-values $<10^{-8}$ ) searches with N (Q40392.1), RPS4 (CAB50708.1) and Ma (CAR94514.1) sequences deprived of LRR, as queries. BlastP (e-values $<30$ ) with PL motifs ${ }^{22}$ were used to retrieve PL domains. All extracted sequence identities were verified using HMMER with default parameters and NB-ARC and PL motifs were aligned to be evaluated.

Sequence alignment, clustering, and phylogenetic reconstruction. Sequence alignments were performed with Muscle ${ }^{62}$ using default parameters and trimmed using MEGA $7^{63}$. Clustering analyses were conducted with CD-HIT ${ }^{64}$ using default parameters and variable sequence identity cut-off values: 0.97 for elimination of redundant sequences (allelic sequences or alternative transcripts) leading the total number of sequences to be reduced to 3734 , and $0.50-0.90$ for the conifer RPW8 domains. To identify RNL groups in conifers, 185 full-length RPW8 sequences ( $>100$ amino acids) were extracted and trimmed from our dataset. These RPW8 domains together with RPW8 domains of ADR1, RPW8.1, RPW8.2 and NRG1 were aligned to undertake the first phylogenetic analyses of conifer sequences. Then, the conifer sequences were clustered using a cut-off value of 0.60 to obtain 45 representative RPW8 sequences. Simultaneously, a second dataset was prepared using 351 Angiosperm, 13 G. biloba and 17 P. abies RNL sequences. These sequences were used to similarly extract full RPW8 domains (>100 amino acids) and clustered (cut-off values: 0.60 ) to obtain 152 representative RPW8 
sequences for this second dataset. The 197 representative sequences were included into the phylogenetic analysis conducted using the web service http://www.phylogeny.fr ${ }^{65}$. The phylogenetic trees were reconstructed using the maximum likelihood (ML) method implemented in the PhyML program (v3.1/3.0 aLRT). The WAG substitution model was selected with an estimated proportion of invariant sites and 4 gamma-distributed rate categories to account for rate heterogeneity across sites. The gamma shape parameter was estimated directly from the data $($ gamma $=3.981$ and gamma $=4.611)$. Reliability for internal branch was assessed using the aLRT test (SH-Like). The phylogenetic trees were then drawn using Dendroscope $3^{66}$. Similarly, the JTT substitution model was used (with Gamma shape parameter: 3.648) resulting in the same tree topology (Supplementary Fig. S8).

Transcriptomic analysis of drought stress in three $P$. glauca clones. Foliage samples from the drought experiment in P. glauca described in Stival Sena et al. ${ }^{40}$ were analysed by RNA-sequencing. The samples were collected at $0,7,14,18$ and 22 days from the beginning of the watering treatments. We used three unrelated and clonally-propagated genotypes (clones 8,11 and 95) each with two replicates, in two conditions (well-watered control and drought with no watering) with five different sampling time points, and obtained 59 RNA samples to analyse (lacking one control replicate for the clone 95 at the time 7 days). The detailed protocol for RNA extraction and RNA-seq library synthesis modified from Stival Sena et al. (2018) ${ }^{40}$ is available in Supplementary Methods. The pooling of 59 libraries into one lane for sequencing produced variable numbers of sequence reads per library (Supplementary Fig. S5). In order to limit the effect of size library, we added a filtering step keeping only genes with counts $>5$ in at least two of the libraries. A principal component analysis (PCA) also showed that replicates clustered mostly together, indicating that plants from the same treatment had similar profiles and suggesting that variation in library size had little impact on expression profiles (Supplementary Fig. S5). DEseq2 v1.20.0 package in $\mathrm{R}$ software (Core Team 2018) was used to normalize count data. We calculated the differential expression between the treatment and the control foliage samples with the DEseq2 v1.20.0 package and the LRT approach. A lists of differentially expressed NLR genes with a FDR adjusted p-value $<0.05$ are shown (Supplementary Table S4). Cluster v2.0.7., factoextra v1.0.5, and pheatmap v1.0.10 packages were used to generate heatmap with gene clustering analyses using manhattan distances and the ward.D2 clustering method ${ }^{67-76}$.

Accession numbers. The raw data from the seven conifer transcriptomes are deposited in the European Nucleotide Archive (ENA) as part of the study SRP134160, bioproject PRJNA437248 (SRA accession SRR6816977 to SRR6816983). A list of accession numbers is available in supplementary methods.

\section{References}

1. Jones, J. D. G. \& Dangl, J. L. The plant immune system. Nature 444, 323-9 (2006).

2. Whitham, S. et al. The product of the tobacco mosaic virus resistance gene N: similarity to toll and the interleukin-1 receptor. Cell 78, 1101-15 (1994).

3. Rossi, M. et al. The nematode resistance gene Mi of tomato confers resistance against the potato aphid. Proc. Natl. Acad. Sci. USA 95, 9750-4 (1998).

4. Claverie, M. et al. The Ma Gene for Complete-Spectrum Resistance to Meloidogyne Species in Prunus Is a TNL with a Huge Repeated C-Terminal Post-LRR Region. Plant Physiol. 156, 779-792 (2011).

5. Ravensdale, M. et al. Intramolecular interaction influences binding of the Flax L5 and L6 resistance proteins to their AvrL567 ligands. PLoS Pathog. 8, e1003004 (2012).

6. Le Roux, C. et al. A receptor pair with an integrated decoy converts pathogen disabling of transcription factors to immunity. Cell 161, 1074-1088 (2015).

7. Chini, A., Grant, J. J., Seki, M., Shinozaki, K. \& Loake, G. J. Drought tolerance established by enhanced expression of the CC-NBSLRR gene, ADR1, requires salicylic acid, EDS1 and ABI1. Plant J. 38, 810-22 (2004).

8. van der Vossen, E. A. G. et al. The Rpi-blb2 gene from Solanum bulbocastanum is an Mi-1 gene homolog conferring broad-spectrum late blight resistance in potato. Plant J. 44, 208-22 (2005).

9. Meyers, B. C., Kozik, A., Griego, A., Kuang, H. \& Michelmore, R. W. Genome-wide analysis of NBS-LRR-encoding genes in Arabidopsis. Plant Cell 15, 809-34 (2003).

10. Xue, J.-Y. et al. A primary survey on bryophyte species reveals two novel classes of nucleotide-binding site (NBS) genes. PLoS One 7 , e36700 (2012).

11. Shao, Z.-Q. et al. Long-term evolution of nucleotide-binding site-leucine-rich repeat genes: understanding gained from and beyond the legume family. Plant Physiol. 166, 217-34 (2014).

12. Jia, Y., Yuan, Y., Zhang, Y., Yang, S. \& Zhang, X. Extreme expansion of NBS-encoding genes in Rosaceae. BMC Genet. 16, 48 (2015).

13. Qian, L.-H. et al. Distinct Patterns of Gene Gain and Loss: Diverse Evolutionary Modes of NBS-Encoding Genes in Three Solanaceae Crop Species. G3 (Bethesda). 7, 1577-1585 (2017).

14. Andolfo, G. et al. Defining the full tomato NB-LRR resistance gene repertoire using genomic and cDNA RenSeq. BMC Plant Biol. 14, $120(2014)$

15. Gao, Y. et al. Out of Water: The Origin and Early Diversification of Plant R-Genes. Plant Physiol. 177, 82-89 (2018).

16. Yue, J., Meyers, B. C., Chen, J., Tian, D. \& Yang, S. Tracing the origin and evolutionary history of plant nucleotide-binding siteleucine-rich repeat (NBS-LRR) genes. New Phytol. 193, 1049-63 (2012).

17. Heath, M. C. Hypersensitive response-related death. Plant Mol. Biol. 44, 321-34 (2000).

18. Moffett, P., Farnham, G., Peart, J. \& Baulcombe, D. C. Interaction between domains of a plant NBS-LRR protein in disease resistancerelated cell death. EMBO J. 21, 4511-4519 (2002).

19. van Ooijen, G. et al. Structure-function analysis of the NB-ARC domain of plant disease resistance proteins. J. Exp. Bot. 59, 1383-97 (2008).

20. McHale, L., Tan, X., Koehl, P. \& Michelmore, R. W. Plant NBS-LRR proteins: adaptable guards. Genome Biol. 7, 212 (2006).

21. Meyers, B. C. et al. Plant disease resistance genes encode members of an ancient and diverse protein family within the nucleotidebinding superfamily. Plant J. 20, 317-32 (1999).

22. Van Ghelder, C. \& Esmenjaud, D. TNL genes in peach: insights into the post-LRR domain. BMC Genomics 17, 317 (2016).

23. Xiao, S. et al. Broad-spectrum mildew resistance in Arabidopsis thaliana mediated by RPW8. Science 291, 118-20 (2001).

24. Zhao, T. et al. A Truncated NLR Protein, TIR-NBS2, Is Required for Activated Defense Responses in the exo70B1 Mutant. PLoS Genet. 11, 1-28 (2015).

25. Nishimura, M. T. et al. TIR-only protein RBA1 recognizes a pathogen effector to regulate cell death in Arabidopsis. Proc. Natl. Acad. Sci. 114, E2053-E2062 (2017). 
26. Zhong, Y. \& Cheng, Z. M. A unique RPW8-encoding class of genes that originated in early land plants and evolved through domain fission. fusion, and duplication. Sci. Rep. 6, 1-13 (2016).

27. Collier, S. M., Hamel, L.-P. \& Moffett, P. Cell death mediated by the N-terminal domains of a unique and highly conserved class of NB-LRR protein. Mol. Plant. Microbe. Interact. 24, 918-31 (2011).

28. Peart, J. R., Mestre, P., Lu, R., Malcuit, I. \& Baulcombe, D. C. NRG1, a CC-NB-LRR protein, together with N, a TIR-NB-LRR protein, mediates resistance against tobacco mosaic virus. Curr. Biol. 15, 968-73 (2005).

29. Bonardi, V. et al. Expanded functions for a family of plant intracellular immune receptors beyond specific recognition of pathogen effectors. Proc. Natl. Acad. Sci. 108, 16463-16468 (2011).

30. Qi, T. et al. NRG1 functions downstream of EDS1 to regulate TIR-NLR-mediated plant immunity in Nicotiana benthamiana. Proc. Natl. Acad. Sci. USA 115, E10979-E10987 (2018).

31. Castel, B. et al. Diverse NLR immune receptors activate defence via the RPW8-NLR NRG1. New Phytol. 222, 966-980 (2019).

32. Meyers, B. C., Morgante, M. \& Michelmore, R. W. TIR-X and TIR-NBS proteins: Two new families related to disease resistance TIRNBS-LRR proteins encoded in Arabidopsis and other plant genomes. Plant J. 32, 77-92 (2002).

33. Liu, J.-J. \& Ekramoddoullah, A. K. M. Isolation, genetic variation and expression of TIR-NBS-LRR resistance gene analogs from western white pine (Pinus monticola Dougl. ex. D. Don.). Mol. Genet. Genomics 270, 432-41 (2003).

34. Liu, J.-J. \& Ekramoddoullah, A. K. M. The CC-NBS-LRR Subfamily in Pinus monticola: Targeted Identification, Gene Expression, and Genetic Linkage with Resistance to Cronartium ribicola. Phytopathology 97, 728-36 (2007).

35. De La Torre, A. R. et al. Insights into conifer giga-genomes. Plant Physiol. 166, 1724-32 (2014).

36. Raherison, E. S. M., Giguère, I., Caron, S., Lamara, M. \& MacKay, J. J. Modular organization of the white spruce (Picea glauca) transcriptome reveals functional organization and evolutionary signatures. New Phytol. 207, 172-87 (2015).

37. Warren, R. L. et al. Improved white spruce (Picea glauca) genome assemblies and annotation of large gene families of conifer terpenoid and phenolic defense metabolism. Plant J. 83, 189-212 (2015).

38. Shao, Z.-Q. et al. Large-Scale Analyses of Angiosperm Nucleotide-Binding Site-Leucine-Rich Repeat Genes Reveal Three Anciently Diverged Classes with Distinct Evolutionary Patterns. Plant Physiol. 170, 2095-109 (2016).

39. Nystedt, B. et al. The Norway spruce genome sequence and conifer genome evolution. Nature 497, 579-84 (2013).

40. Stival Sena, J., Giguère, I., Rigault, P., Bousquet, J. \& Mackay, J. Expansion of the dehydrin gene family in the Pinaceae is associated with considerable structural diversity and drought-responsive expression. Tree Physiol. 38, 442-456 (2018).

41. Pavy, N. et al. A high-resolution reference genetic map positioning $8.8 \mathrm{~K}$ genes for the conifer white spruce: structural genomics implications and correspondence with physical distance. Plant J. 90, 189-203 (2017).

42. Danot, O., Marquenet, E., Vidal-Ingigliardi, D. \& Richet, E. Wheel of Life, Wheel of Death: A Mechanistic Insight into Signaling by STAND Proteins. Structure 17, 172-82 (2009).

43. Chini, A. \& Loake, G. J. Motifs specific for the ADR1 NBS-LRR protein family in Arabidopsis are conserved among NBS-LRR sequences from both dicotyledonous and monocotyledonous plants. Planta 221, 597-601 (2005).

44. Williams, S. J. et al. An Autoactive Mutant of the M Flax Rust Resistance Protein Has a Preference for Binding ATP, Whereas WildType M Protein Binds ADP. Mol. Plant-Microbe Interact. 24, 897-906 (2011).

45. Bernoux, M. et al. Comparative Analysis of the Flax Immune Receptors L6 and L7 Suggests an Equilibrium-Based Switch Activation Model. Plant Cell 28, 146-59 (2016).

46. Urbach, J. M. \& Ausubel, F. M. The NBS-LRR architectures of plant R-proteins and metazoan NLRs evolved in independent events. Proc. Natl. Acad. Sci. USA 114, 1063-1068 (2017).

47. Savard, L., Li, P., Strausst, S. H., Chase, M. W. \& Michaud, M. Chloroplast and nuclear gene sequences indicate Late Pennsylvanian time for the last common ancestor of extant seed plants. 91, 5163-5167 (1994).

48. Klutsch, J. G., Shamoun, S. F. \& Erbilgin, N. Drought stress leads to systemic induced susceptibility to a nectrotrophic fungus associated with mountain pine beetle in Pinus banksiana seedlings. PLoS One 12, e0189203 (2017).

49. Ryu, M., Mishra, R. C., Jeon, J., Lee, S. K. \& Bae, H. Drought-induced susceptibility for Cenangium ferruginosum leads to progression of Cenangium-dieback disease in Pinus koraiensis. Sci. Rep. 8, 1-14 (2018).

50. Trujillo-Moya, C. et al. Drought Sensitivity of Norway Spruce at the Species' Warmest Fringe: Quantitative and Molecular Analysis Reveals High Genetic Variation Among and Within Provenances. G3 (Bethesda). 8, 1225-1245 (2018).

51. Rathjen, J. P. \& Dodds, P. N. Dancing with the Stars: An Asterid NLR Family. Trends Plant Sci. 22, 1003-1005 (2017).

52. Xiao, S. et al. The atypical resistance gene, RPW8, recruits components of basal defence for powdery mildew resistance in Arabidopsis. Plant J. 42, 95-110 (2005).

53. Lapin, D. et al. A coevolved EDS1-SAG101-NRG1 module mediates cell death signaling by TIR-domain immune receptors. Biorxiv, https://doi.org/10.1101/572826 (2019)

54. Baggs, E. et al. Convergent gene loss in aquatic plants predicts new components of plant immunity and drought response. Biorxiv, https://doi.org/10.1101/572560 (2019).

55. Wu, C.-H. et al. NLR network mediates immunity to diverse plant pathogens. Proc. Natl. Acad. Sci. USA 114, 8113-8118 (2017)

56. Raherison, E. et al. Transcriptome profiling in conifers and the PiceaGenExpress database show patterns of diversification within gene families and interspecific conservation in vascular gene expression. BMC Genomics 13, 434 (2012).

57. Chang, S., Puryear, J. \& Cairney, J. A simple and efficient method for isolating RNA from pine trees. Plant Mol. Biol. Report. 11, $113-116(1993)$

58. Pavy, N. et al. Identification of conserved core xylem gene sets: Conifer cDNA microarray development, transcript profiling and computational analyses. New Phytol. 180, 766-786 (2008).

59. Rigault, P. et al. A White Spruce Gene Catalog for Conifer Genome Analyses. Plant Physiol. 157, 14-28 (2011).

60. Finn, R. D. et al. HMMER web server: 2015 update. Nucleic Acids Res. 43, W30-8 (2015).

61. Bailey, T. L. et al. MEME SUITE: tools for motif discovery and searching. Nucleic Acids Res. 37, W202-8 (2009)

62. Edgar, R. C. MUSCLE: multiple sequence alignment with high accuracy and high throughput. Nucleic Acids Res. 32, 1792-7 (2004).

63. Kumar, S., Stecher, G. \& Tamura, K. MEGA7: Molecular Evolutionary Genetics Analysis Version 7.0 for Bigger Datasets. Mol. Biol. Evol. 33, 1870-4 (2016).

64. Li, W. \& Godzik, A. Cd-hit: a fast program for clustering and comparing large sets of protein or nucleotide sequences. Bioinformatics 22, 1658-9 (2006).

65. Dereeper, A., Audic, S., Claverie, J.-M. \& Blanc, G. BLAST-EXPLORER helps you building datasets for phylogenetic analysis. BMC Evol. Biol. 10, 8 (2010).

66. Huson, D. H. \& Scornavacca, C. Dendroscope 3: an interactive tool for rooted phylogenetic trees and networks. Syst. Biol. 61, 1061-7 (2012).

67. Zhang, J. et al. Receptor-like cytoplasmic kinases integrate signaling from multiple plant immune receptors and are targeted by a Pseudomonas syringae effector. Cell Host Microbe 7, 290-301 (2010).

68. Jupe, F. et al. Identification and localisation of the NB-LRR gene family within the potato genome. BMC Genomics 13, 75 (2012)

69. Kale, S. M., Pardeshi, V. C., Barvkar, V. T., Gupta, V. S. \& Kadoo, N. Y. Genome-wide identification and characterization of nucleotide binding site leucine-rich repeat genes in linseed reveal distinct patterns of gene structure. Genome 56, 91-9 (2013).

70. Kohler, A. et al. Genome-wide identification of NBS resistance genes in Populus trichocarpa. Plant Mol. Biol. 66, 619-36 (2008).

71. Wan, H. et al. Genome-wide analysis of NBS-encoding disease resistance genes in Cucumis sativus and phylogenetic study of NBSencoding genes in Cucurbitaceae crops. BMC Genomics 14, 109 (2013). 
72. Porter, B. W. et al. Genome-wide analysis of Carica papaya reveals a small NBS resistance gene family. Mol. Genet. Genomics 281, 609-26 (2009).

73. Yang, S., Zhang, X., Yue, J.-X., Tian, D. \& Chen, J.-Q. Recent duplications dominate NBS-encoding gene expansion in two woody species. Mol. Genet. Genomics 280, 187-98 (2008).

74. Mun, J.-H., Yu, H.-J., Park, S. \& Park, B.-S. Genome-wide identification of NBS-encoding resistance genes in Brassica rapa. Mol. Genet. Genomics 282, 617-31 (2009).

75. Guo, Y.-L. et al. Genome-Wide Comparison of Nucleotide-Binding Site-Leucine-Rich Repeat-Encoding Genes in Arabidopsis. Plant Physiol. 157, 757-769 (2011).

76. Kang, Y. J. et al. Genome-wide mapping of NBS-LRR genes and their association with disease resistance in soybean. BMC Plant Biol. 12, 139 (2012).

\section{Acknowledgements}

We thank F. Larochelle and M.-A Paré for assistance with plant procurement and growth, S. Laviolette and S. Senneville for project management, and K. Guay, K. Beaupré-Boivin, D. Miclette and A. Azaiez for sample collections and processing. This study was developed in part as a collaboration with the ProCoGen project (https://bfw.ac.at/rz/bfwcms2.web?dok=9020) (funded by the European Union's Seventh Framework Programme). This work was supported by a research grant from the Québec Ministry for the Economy, Science and Innovation, program for the support of international initiatives to $\mathrm{AD}$, JB and PR. CVG was supported by the Plant - Nematode Interaction team, at ISA-INRA in Sophia Antipolis, France. JM is supported by M. and A. Wood and by the P. Wood Trust.

\section{Author Contributions}

J.M., P.R., J.P., G.J.P., A.D. and J.B. designed the transcriptome research, I.G. and S.C. carried out and supervised the field and laboratory work, P.R. analysed the RNA-Seq data, developed the reference transcriptomes and did the data releases, J.S.S. planned and carried out the drought responsiveness experiment and G.J.P. performed the related RNA-Seq data analysis, C.V.G., J.M. and D.E. planned and designed the NLR analyses, C.V.G. developed and analysed the NLR data and drafted the manuscript, C.V.G. and J.M. revised the manuscript, all of the authors commented and approved the manuscript.

\section{Additional Information}

Supplementary information accompanies this paper at https://doi.org/10.1038/s41598-019-47950-7.

Competing Interests: The authors declare no competing interests.

Publisher's note: Springer Nature remains neutral with regard to jurisdictional claims in published maps and institutional affiliations.

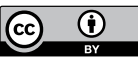

Open Access This article is licensed under a Creative Commons Attribution 4.0 International License, which permits use, sharing, adaptation, distribution and reproduction in any medium or format, as long as you give appropriate credit to the original author(s) and the source, provide a link to the Creative Commons license, and indicate if changes were made. The images or other third party material in this article are included in the article's Creative Commons license, unless indicated otherwise in a credit line to the material. If material is not included in the article's Creative Commons license and your intended use is not permitted by statutory regulation or exceeds the permitted use, you will need to obtain permission directly from the copyright holder. To view a copy of this license, visit http://creativecommons.org/licenses/by/4.0/.

(C) The Author(s) 2019 